\title{
A study of smoothed TEC precision inferred from GPS measurements
}

\author{
Zhizhao Liu, Yang Gao, and Susan Skone \\ Department of Geomatics Engineering, The University of Calgary, 2500 University Drive N.W., Calgary, Alberta, Canada, T2N 1N4
}

(Received February 23, 2005; Revised July 19, 2005; Accepted July 21, 2005)

\begin{abstract}
The availability of a large amount of TEC data derived from dual frequency GPS measurements observed by GPS reference networks provides a great opportunity for ionosphere studies. In order to obtain better accuracy for the derived TEC, a data smoothing technique is usually employed to take advantage of both code pseudorange and carrier phase GPS measurements. The precision of TEC data therefore is dependent on the smoothing approach. However little work has been done to evaluate the precision of the smoothed TEC data obtained from different smoothing approaches. This investigation examines the properties of two popularly used smoothing approaches and develops the closed-form formulas for estimating the precision of the smoothed TEC data. In addition, a previously proposed approximate formula for estimating TEC precision is also evaluated against its closed-form formula developed in this paper. The TEC precisions derived from the closed-form precision estimation formulas for approaches I and II are analyzed in a numerical test. The results suggest that approach II outperforms approach I and the precision of TEC data smoothed by approach II is higher than approach I. For approach I, a numerical test is also conducted to compare the precision difference between the closed-form and approximate formulas for estimating TEC precision. The comparison indicates that TEC derived from the closed-form formula have better precisions than the approximate formula. Analysis also reminds users that extra cautions should be taken when using the approximate formula in order to avoid the precision divergence phenomenon.
\end{abstract}

Key words: Global Positioning System, total electron content, data smoothing, precision estimation.

\section{Introduction}

The total electron content (TEC) can be easily inferred from dual frequency Global Positioning System (GPS) measurements using the refractivity property of the ionosphere (Sardon et al., 1994; Leick, 1995). To date, a number of regional and global GPS reference networks have been deployed by different organizations worldwide. These networks are typically equipped with high quality dual frequency GPS receivers and operate in an uninterrupted mode for continuous data acquisition. Dual frequency GPS measurements are real-time recorded and distributed to users for various types of research and application purposes. At present, there are more than 1000 reference stations that are equipped with dual frequency GPS receivers and are continuously operating (Liu et al., 2005). Considering that each GPS receiver can track an average of 10 satellites at one time, about 10 TEC measurements can be derived from each receiver at every epoch. A huge number of TEC observations can be retrieved using the worldwide GPS networks. This type of GPS-derived TEC data has become increasingly important to ionosphere studies and they have been extensively used as a source of input data for ionospheric modeling and space weather monitoring, e.g. Hajj et al. (1994), Hernández-Pajares et al. (1999, 2002), Langley et al. (2002).

Dual frequency GPS receivers can record both code pseudorange and carrier phase measurements on L1 and L2 fre-

Copyright (c) The Society of Geomagnetism and Earth, Planetary and Space Sciences (SGEPSS); The Seismological Society of Japan; The Volcanological Society of Japan; The Geodetic Society of Japan; The Japanese Society for Planetary Sciences; TERRAPUB quencies. Code pseudorange is an unambiguous distance measurement between a GPS satellite and a GPS receiver. It can be used to directly derive an absolute TEC estimate with an accuracy of 1-5 TECU. The code-derived TEC is subject to high noise and multipath effects in the pseudorange measurements (Skone et al., 2002). As for the multipath effects in the TEC data, when proper GPS receiver and antenna as well as observation environment is appropriately selected, the multipath effect on GPS measurements can be significantly mitigated. The multipath effect in this investigation is assumed to be at negligible level and is not taken into account in the following equation derivations. But for the noise level of the code-derived TEC data, it is much higher than the carrier-phase-derived data. The carrierphase-derived TEC estimates have a much higher accuracy than the code-derived TEC estimates but they are ambiguous due to the existence of integer phase ambiguities in the carrier phase measurements. Due to the difficulty in ambiguity determination, a technique known as data smoothing has been widely used to combine both code and carrier phase observations to improve TEC estimate accuracy. Nowadays some agencies estimate TEC data from GPS carrier phase measurements after resolving the carrier phase ambiguities when processing data from a network of GPS stations for station coordinate determination. Correspondingly the purely carrier phase-based TEC estimates should be more accurate than the carrier phase smoothed TEC estimates if the ambiguities are correctly resolved. However, the ambiguity resolution will become more difficult and less reliable when the baselines between GPS stations are long and severe ionospheric conditions are present. In addition, 
in most cases the ambiguity resolution is usually resolved by forming baselines between different GPS stations in a network. In comparison, the carrier phase smoothing codederived TEC data can be performed on a single GPS station instead of forming baselines with other stations. This is very convenient when expanding the GPS network size by including more GPS stations, because the TEC derivation on each GPS station is independent of each other. On the contrast, both the complexity and the computational burden of deriving purely carrier phase-based TEC data from GPS network ambiguity resolution is significantly higher than the carrier phase smoothing approach. This will become more apparent when more GPS stations are included in the GPS network for processing. Therefore, the approach of using carrier phase to smooth code-derived TEC data is one of widely used methods to obtain high accuracy TEC data with relatively simple computation procedures.

To date several algorithms have been developed to perform smoothing for the derivation of TEC data and their implementations typically consist of two different approaches. One approach is to first perform the smoothing on raw GPS measurements and then use the smoothed GPS data to estimate TEC (Hatch, 1982; Lachapelle et al., 1986; Liu and Gao, 2001). The other approach, opposite to the former, is first to derive TEC from raw GPS measurements and then smooth the derived TEC data (Skone, 1998; Liu, 2004). The former approach is thereafter referred to as approach I and the latter as approach II. In the smoothing approach I, one should note that during the carrier phase smoothing pseudorange, a smoothing weight factor has to be appropriately determined before the commencement of the smoothing and that a divergence may occur when smoothing is performed over a long period of time because of the ionospheric divergence character between the carrier phase and pseudorange measurements. In comparison, the smoothing approach II does not have similar problems during the smoothing. The determination of the precision of the smoothed TEC data is important because the precision information is necessary for forming the variance-covariance matrix for the TEC data in least-squares or Kalman filter estimation when the derived TEC data are used for other purposes, e.g. studying the ionosphere and space position determination (Sekido et al., 2003). However, little attention has so far been paid to the precision estimation of smoothed TEC data as well as the examination of the precision difference between the two smoothing approaches. This paper will center on the subject of estimating the precision of smoothed TEC data and comparing the precision difference of two approaches.

The paper will first derive the closed-form formulas for precision computation of GPS-derived TEC data for both approach I and II, after briefly reviewing the two often used smoothing approaches. In section three, a function is defined in order to compare the precision differences of TEC data obtained from the two approaches. The numerical analysis is conducted to examine the performance of the two approaches. In section four, an approximate formula used for estimating TEC precision for approach I is also evaluated against its closed-form formula and their evaluation results from a numerical analysis are presented. In the conclusion, the performance and smoothing efficiency of the studied smoothing approaches will be summarized.

\section{Two Smoothing Approaches}

Various smoothing approaches have been developed in the past decades to improve the precision of TEC data derived from GPS measurements (Hatch, 1982; Lachapelle et al., 1986; Jin, 1996; Skone, 1998; Liu, 2004). Compared to the TEC data not being smoothed (i.e. directly derived from GPS code pseudoranges), the smoothed TEC data typical have an improvement of a few TECU in precision.

\subsection{Smoothing approach $I$}

A widely used algorithm to smooth the code pseudorange with carrier phase measurements was initially proposed in Hatch (1982). This algorithm is further improved to introduce an epoch-dependent smoothing weight factor (SWF) (Hatch, 1986). A modification is further made to this algorithm by reducing the SWF by a constant value with the increase of number of GPS data epoch used for smoothing (Lachapelle et al., 1986). The carrier phase smoothing code pseudorange approach is described as follows:

$$
\begin{aligned}
R\left(t_{i}\right)_{\mathrm{sm}}= & w_{i} R\left(t_{i}\right) \\
& +\left(1-w_{i}\right)\left[R\left(t_{i-1}\right)_{\mathrm{sm}}+\Phi\left(t_{i}\right)-\Phi\left(t_{i-1}\right)\right]
\end{aligned}
$$

where $R\left(t_{i}\right)$ and $\Phi\left(t_{i}\right)$ are the code pseudorange and carrier phase measurements at epoch $t_{i}$, respectively (note that the carrier phase measurement has been converted into range with a distance unit of meter); $w_{i}$ is the smoothing weight factor with an initial value of 1 and varying within [0 1]; $R\left(t_{i}\right)_{\text {sm }}$ is the resultant smoothed code measurement at epoch $t_{i}$. In this investigation, two computational experiments are conducted. One experiment allows SWF to decrease from 1.0 with a decrement scale of 0.010 each epoch in the smoothing process and the other one allows SWF to start from 1.0 with a downscale of 0.005 with the introduction of a new epoch in the smoothing.

In order to evaluate the accuracy of the smoothed code pseudorange measurement, the expanded analytical form of Eq. (1) has to be developed. Starting from the initial condition of $R\left(t_{1}\right)_{\mathrm{sm}}=w_{1} R\left(t_{1}\right)$ at the first epoch, the following expanded expression can be derived for the smoothed code measurement at epoch $t_{i}$.

$$
\begin{aligned}
R\left(t_{i}\right)_{\mathrm{sm}}= & w_{1}\left(1-w_{2}\right) \cdots\left(1-w_{i}\right) R\left(t_{1}\right) \\
& +w_{2}\left(1-w_{3}\right) \cdots\left(1-w_{i}\right) R\left(t_{2}\right)+\cdots+w_{i} R\left(t_{i}\right) \\
& -w_{1}\left(1-w_{2}\right) \cdots\left(1-w_{i}\right) \Phi\left(t_{1}\right) \\
& -w_{2}\left(1-w_{3}\right) \cdots\left(1-w_{i}\right) \Phi\left(t_{2}\right) \\
& -\cdots-w_{i-1}\left(1-w_{i}\right) \Phi\left(t_{i-1}\right) \\
& -w_{i} \Phi\left(t_{i}\right)+\Phi\left(t_{i}\right) \\
= & \Phi\left(t_{i}\right)+\sum_{k=1}^{i} w_{k}\left(1-w_{k+1}\right)\left(1-w_{k+2}\right) \cdots\left(1-w_{i}\right) \\
& \times\left[R\left(t_{k}\right)-\Phi\left(t_{k}\right)\right]
\end{aligned}
$$

Assuming that the code pseudorange and carrier phase measurements are uncorrelated and that measurements are uncorrelated between two consecutive epochs, the general formula to calculate the standard deviation for the smoothed code pseudorange measurements can be obtained from 
Eq. (2) as follows:

$$
\begin{aligned}
\sigma_{R\left(t_{i}\right)_{\mathrm{sm}}}^{2}= & \left(1-2 w_{i}\right) \sigma_{\Phi\left(t_{i}\right)}^{2} \\
& +\sum_{k=1}^{i}\left[w_{k}\left(1-w_{k+1}\right)\left(1-w_{k+2}\right) \cdots\left(1-w_{i}\right)\right]^{2} \\
& \times\left(\sigma_{R\left(t_{k}\right)}^{2}+\sigma_{\Phi\left(t_{k}\right)}^{2}\right)
\end{aligned}
$$

where $\sigma_{R\left(t_{i}\right)_{\mathrm{sm}}}$ is the standard deviation for the smoothed code pseudorange; and $\sigma_{R\left(t_{k}\right)}$ and $\sigma_{\Phi\left(t_{k}\right)}$ are standard deviations for the raw code pseudorange carrier phase measurements at epoch $t_{k}$, respectively. Let $\sigma_{R}^{2}=S \sigma_{\Phi}^{2}$, Eq. (3) can be simplified as:

$$
\begin{aligned}
\sigma_{R\left(t_{i}\right)_{\mathrm{sm}}}^{2}= & \left(1-2 w_{i}\right) \sigma_{\Phi\left(t_{i}\right)}^{2} \\
& +(S+1) \sum_{k=1}^{i}\left[w_{k}\left(1-w_{k+1}\right)\left(1-w_{k+2}\right)\right. \\
& \left.\times\left(1-w_{k+3}\right) \cdots\left(1-w_{i}\right)\right]^{2} \sigma_{\Phi\left(t_{k}\right)}^{2}
\end{aligned}
$$

Assuming that $\sigma_{\Phi\left(t_{1}\right)}^{2}=\sigma_{\Phi\left(t_{2}\right)}^{2}=\cdots=\sigma_{\Phi\left(t_{k}\right)}^{2}=\cdots=\sigma_{\Phi}^{2}$, Eq. (4) becomes:

$$
\begin{aligned}
& \sigma_{R\left(t_{i}\right)_{\mathrm{sm}}}^{2}=\sigma_{\Phi}^{2}\left\{\left(1-2 w_{i}\right)+(S+1) \sum_{k=1}^{i}\left[w_{k}\left(1-w_{k+1}\right)\right.\right. \\
& \left.\left.\times\left(1-w_{k+2}\right) \cdots\left(1-w_{i}\right)\right]^{2}\right\}
\end{aligned}
$$

As stated before, once the smoothed code pseudorange measurements at L1 and L2 frequencies are obtained, they can be used to derive ionospheric total electron content as follows (Liu and Gao, 2001):

$$
\operatorname{TEC}\left(t_{i}\right)=\frac{f_{1}^{2}\left(R\left(t_{i}\right)_{1, \mathrm{sm}}-R\left(t_{i}\right)_{2, \mathrm{sm}}-B_{i}-B^{p}\right)}{40.3 \times 10^{16}(1-\gamma)}
$$

where TEC $\left(t_{i}\right)$ is the total electron content at epoch $t_{i}$ in the unit of TECU (total electron content unit); $R\left(t_{i}\right)_{1, \mathrm{sm}}$ and $R\left(t_{i}\right)_{2, \text { sm }}$ are the smoothed code pseudorange measurements for L1 and L2 frequencies that are derived using Eq. (2); $B_{i}$ and $B^{p}$ are the GPS receiver and satellite inter-frequency biases for the code measurements, respectively; $\gamma$ is the squared ratio of L1 and L2 frequencies, $\gamma=\left(f_{1} / f_{2}\right)^{2}=$ $(1575.42 / 1227.6)^{2}=(77 / 60)^{2}$. Thus the standard deviation of TEC $\left(t_{i}\right)$, denoted as $\sigma_{\mathrm{TEC}\left(t_{i}\right)}$, can be calculated from Eq. (6) as $\frac{f_{1}^{2}}{40.3 \times 10^{16}(\gamma-1)} \sqrt{\sigma_{R\left(t_{i}\right)_{1, \mathrm{sm}}}^{2}+\sigma_{R\left(t_{i}\right)_{2, \mathrm{sm}}}^{2}}$ considering the fact that the inter-frequency biases can be determined with an accuracy of $0.1 \mathrm{~ns}$ for satellite inter-frequency bias and of $1 \mathrm{~ns}$ for receiver inter-frequency bias by IGS Analysis Centers. In this study, it is assumed that it is a good approximation to treat the biases as known parameters thus the contribution to standard deviation errors from interfrequency biases is not considered. To be simple, assuming that $\sigma_{R\left(t_{i}\right)_{1, \mathrm{sm}}}=\sigma_{R\left(t_{i}\right)_{2, \mathrm{sm}}}$ (the noise on P2 pseudorange code measurement might be actually larger than that on P1 due to the activation of antispoof), the expression to calculate $\sigma_{\mathrm{TEC}\left(t_{i}\right)}$ can be further simplified as $\frac{\sqrt{2} f_{1}^{2}}{40.3 \times 10^{16}(\gamma-1)} \sigma_{R\left(t_{i}\right)_{1, \mathrm{sm}}}$. Taking Eq. (5) into account, one has:

$$
\sigma_{\mathrm{TEC}\left(t_{i}\right)}=\frac{\sqrt{2} f_{1}^{2} \sigma_{\Phi}}{40.3 \times 10^{16}(\gamma-1)}
$$

$$
\begin{aligned}
& \times\left\{\left(1-2 w_{i}\right)+(S+1) \sum_{k=1}^{i}\left[w_{k}\left(1-w_{k+1}\right)\right.\right. \\
& \left.\left.\left(1-w_{k+2}\right)\left(1-w_{k+3}\right) \cdots\left(1-w_{i}\right)\right]^{2}\right\}^{1 / 2}
\end{aligned}
$$

In Eq. (7), both $\gamma$ and $f_{1}$ are constants; $S$ is a predetermined parameter that is dependent on the noise level of both code pseudorange and carrier phase measurements; the value of $w_{k}(k=1,2, \cdots i)$ is the epoch-dependent SWF and has been defined prior to the start of the smoothing process. The precision of the smoothed $\operatorname{TEC}\left(t_{i}\right)$ data at any epoch $t_{i}(i=1,2, \cdots)$ can be estimated using Eq. (7).

\subsection{Smoothing approach II}

The smoothing approach II takes a strategy different from approach I. It first derives TEC data from both code pseudorange and carrier phase measurements and then performs the smoothing process. In this approach, the code pseudorange derived TEC data, denoted as $\operatorname{TEC}\left(t_{i}\right)_{R}$, and the carrier phase derived TEC data, represented by $\operatorname{TEC}\left(t_{i}\right)_{\Phi}$, are first inferred from dual frequency GPS measurements using the formulas below.

$$
\begin{aligned}
& \operatorname{TEC}\left(t_{i}\right)_{R}=\frac{f_{1}^{2}\left[\left(R\left(t_{i}\right)_{1}-R\left(t_{i}\right)_{2}\right)-B_{i}-B^{p}\right]}{40.3 \times 10^{16}(1-\gamma)} \\
& \operatorname{TEC}\left(t_{i}\right)_{\Phi} \\
& =\frac{f_{1}^{2}\left[\left(\Phi\left(t_{i}\right)_{1}-\Phi\left(t_{i}\right)_{2}\right)-\left(\lambda_{1} N_{1}-\lambda_{2} N_{2}\right)-b_{i}-b^{p}\right]}{40.3 \times 10^{16}(\gamma-1)}
\end{aligned}
$$

Equation (8) is very similar to Eq. (6) except that the raw GPS observations are used here instead of smoothed code pseudoranges. In Eq. (9), $\Phi\left(t_{i}\right)_{1}$ and $\Phi\left(t_{i}\right)_{2}$ are the carrier phase measurements at epoch on L1 and L2 frequencies, respectively; $N_{1}$ and $N_{2}$ are the ambiguities for $\Phi\left(t_{i}\right)_{1}$ and $\Phi\left(t_{i}\right)_{2}$, respectively; $\lambda_{1}$ and $\lambda_{2}$ are wavelength of L1 and L2 frequencies, respectively; $b_{i}$ and $b^{p}$ are the GPS receiver and satellite inter-frequency biases for carrier phase measurement, respectively.

Differencing $\operatorname{TEC}\left(t_{i}\right)_{R}$ with respect to $\operatorname{TEC}\left(t_{i}\right)_{\Phi}$, the resultant difference, denoted as $\triangle \mathrm{TEC}\left(t_{i}\right)$, is usually referred to as TEC offset. This TEC offset can be computed at each epoch by:

$$
\begin{aligned}
& \Delta \operatorname{TEC}\left(t_{i}\right)=\operatorname{TEC}\left(t_{i}\right)_{R}-\operatorname{TEC}\left(t_{i}\right)_{\Phi} \\
& =\frac{f_{1}^{2}\left[\left(R\left(t_{i}\right)_{1}-R\left(t_{i}\right)_{2}\right)-B_{i}-B^{p}+\left(\Phi\left(t_{i}\right)_{1}-\Phi\left(t_{i}\right)_{2}\right)\right]}{40.3 \times 10^{16}(1-\gamma)} \\
& -\frac{f_{1}^{2}\left[-\left(\lambda_{1} N_{1}-\lambda_{2} N_{2}\right)-b_{i}-b^{p}\right]}{40.3 \times 10^{16}(\gamma-1)}
\end{aligned}
$$

At each epoch, one $\Delta \operatorname{TEC}\left(t_{i}\right)(i=1,2, \cdots n)$ can be calculated provided that dual frequency GPS observations are available. Theoretically, $\Delta \operatorname{TEC}\left(t_{i}\right)(i=1,2, \cdots n)$ should be constant over time as long as the GPS signals are continuously tracked and no cycle slip occurs (i.e. carrier phase ambiguities do not change over time) because both TEC $\left(t_{i}\right)_{R}$ and $\operatorname{TEC}\left(t_{i}\right)_{\Phi}$ are the measurements of the same total electron content over the same location for the same epoch. The only difference between TEC $\left(t_{i}\right)_{R}$ and $\operatorname{TEC}\left(t_{i}\right)_{\Phi}$ is that there are two ambiguities in $\operatorname{TEC}\left(t_{i}\right)_{\Phi}$, as 
indicated in Eq. (9). The two ambiguities are constant over time if GPS signals are continuously tracked and no cycle slips are present. For each pair of satellites and receivers, one $\triangle \mathrm{TEC}\left(t_{i}\right)$ can be derived at each epoch. A more precise TEC offset for epoch $t_{i}$, denoted as $\triangle \mathrm{TEC}\left(t_{i}\right)_{\mathrm{sm}}$, can be obtained if all the TEC offsets prior to epoch $t_{i}$ are employed to perform the smoothing. A recursive estimation algorithm has been developed to estimate a more precise TEC offset for epoch $t_{i}$ as shown below (Skone, 1998).

$$
\begin{aligned}
\Delta \operatorname{TEC}\left(t_{i}\right)_{\mathrm{sm}}= & \frac{i-1}{i} \Delta \operatorname{TEC}\left(t_{i-1}\right)_{\mathrm{sm}} \\
& +\frac{1}{i}\left(\operatorname{TEC}\left(t_{i}\right)_{R}-\operatorname{TEC}\left(t_{i}\right)_{\Phi}\right)
\end{aligned}
$$

This smoothed offset $\triangle \mathrm{TEC}\left(t_{i}\right)_{\mathrm{sm}}$ is then added up to the carrier phase derived TEC data to obtain the absolute TEC data. The resultant TEC, denoted as TEC $\left(t_{i}\right)_{\mathrm{sm}}$, thus possesses the feature of both absoluteness and smoothness.

$$
\begin{aligned}
\operatorname{TEC}\left(t_{i}\right)_{\mathrm{sm}}= & \operatorname{TEC}\left(t_{i}\right)_{\Phi}+\Delta \operatorname{TEC}\left(t_{i}\right)_{\mathrm{sm}} \\
= & \frac{1}{i} \sum_{k=1}^{i} \operatorname{TEC}\left(t_{k}\right)_{R}-\frac{1}{i} \sum_{k=1}^{i-1} \operatorname{TEC}\left(t_{k}\right)_{\Phi} \\
& +\frac{i-1}{i} \operatorname{TEC}\left(t_{i}\right)_{\Phi}
\end{aligned}
$$

The standard deviation of TEC $\left(t_{i}\right)_{\mathrm{sm}}$, denoted as $\sigma_{\mathrm{TEC}\left(t_{i}\right)_{\mathrm{sm}}}$ can be derived from Eq. (12) as below assuming that $\operatorname{TEC}\left(t_{i}\right)_{R}$ and $\operatorname{TEC}\left(t_{i}\right)_{\Phi}$ are uncorrelated with each other and that the TEC data of consecutive epochs are uncorrelated as well.

$$
\sigma_{\mathrm{TEC}\left(t_{i}\right)_{\mathrm{sm}}}=\sqrt{\frac{1}{i} \sigma_{\mathrm{TEC}\left(t_{k}\right)_{R}}^{2}+\frac{i-1}{i} \sigma_{\mathrm{TEC}\left(t_{k}\right)_{\Phi}}^{2}}
$$

where $\sigma_{\mathrm{TEC}\left(t_{k}\right)_{R}}$ and $\sigma_{\mathrm{TEC}\left(t_{k}\right)_{\Phi}}$ are the standard deviations for TEC data derived from code pseudorange and carrier phase measurements, respectively. They can be derived from Eqs. (8) and (9), respectively:

$$
\begin{aligned}
\sigma_{\mathrm{TEC}\left(t_{k}\right)_{R}} & =\frac{f_{1}^{2}}{40.3 \times 10^{16}(\gamma-1)} \sqrt{\sigma_{R\left(t_{k}\right)_{1}}^{2}+\sigma_{R\left(t_{k}\right)_{2}}^{2}} \\
\sigma_{\mathrm{TEC}\left(t_{k}\right)_{\Phi}} & =\frac{f_{1}^{2}}{40.3 \times 10^{16}(\gamma-1)} \sqrt{\sigma_{\Phi\left(t_{k}\right)_{1}}^{2}+\sigma_{\Phi\left(t_{k}\right)_{2}}^{2}}
\end{aligned}
$$

Inserting Eqs. (14) and (15) into (13), the resultant $\sigma_{\mathrm{TEC}\left(t_{i}\right)_{\mathrm{sm}}}$ can be written as follows:

$$
\begin{aligned}
& \sigma_{\mathrm{TEC}\left(t_{i}\right)_{\mathrm{sm}}}=\frac{f_{1}^{2}}{40.3 \times 10^{16}(\gamma-1) \sqrt{i}} \\
& \times \sqrt{\sigma_{R\left(t_{k}\right)_{1}}^{2}+\sigma_{R\left(t_{k}\right)_{2}}^{2}+(i-1) \sigma_{\Phi\left(t_{k}\right)_{1}}^{2}+(i-1) \sigma_{\Phi\left(t_{k}\right)_{2}}^{2}}
\end{aligned}
$$

As stated before, the noise level of the code pseudorange measurement is approximately $\sqrt{S}$ times of the carrier phase one. If assume $\sigma_{R\left(t_{k}\right)_{1}}=\sigma_{R\left(t_{k}\right)_{2}}$ and $\sigma_{\Phi\left(t_{k}\right)_{1}}=\sigma_{\Phi\left(t_{k}\right)_{2}}$, a simplified expression of calculating standard deviation $\sigma_{\mathrm{TEC}}\left(t_{i}\right)_{\mathrm{sm}}$ for smoothed TEC data is obtained as follows:

$$
\sigma_{\mathrm{TEC}\left(t_{i}\right)_{\mathrm{sm}}}=\frac{\sqrt{2} f_{1}^{2} \sigma_{\Phi\left(t_{k}\right)}}{40.3 \times 10^{16}(\gamma-1)} \sqrt{\frac{S+i-1}{i}}
$$

\section{Comparison of the Two Smoothing Approaches}

The above section presents the development of the formulas to estimate standard deviations for two different TEC smoothing approaches. The two formulas are given in Eqs. (7) and (17), respectively. Both approaches employ a recursive algorithm to realize real-time derivation of TEC data on the basis of making use of all GPS observations at previous epochs. Therefore, there is no significant difference between the two approaches in terms of the computational efficiency. In particular, the current advanced computing technology makes the difference in the computational efficiency to a minimum level. On the contrary, the accuracy of the derived TEC data is by far a more concerned subject than computation efficiency.

In order to perform a comparison of the accuracies obtained from the two smoothing approaches, a function $F(i)$ is defined as the subtraction of the standard deviation of approach I with respect to that of approach II:

$$
\begin{aligned}
& F(i)=\sigma_{\operatorname{TEC}\left(t_{i}\right)}-\sigma_{\operatorname{TEC}\left(t_{i}\right) \mathrm{sm}} \\
& =\frac{\sqrt{2} f_{1}^{2} \sigma_{\Phi\left(t_{1}\right)}}{40.3 \times 10^{16}(\gamma-1)} \\
& \times\left(\sqrt{\left(1-2 w_{i}\right)+(S+1) \sum_{k=1}^{i}\left[w_{k}\left(1-w_{k+1}\right) \cdots\left(1-w_{i}\right)\right]^{2}}\right. \\
& \left.-\sqrt{\frac{S+i-1}{i}}\right)
\end{aligned}
$$

The value of function $F(i)$ depends on the number of epochs that are used in the smoothing practice since other parameters such as $f_{1}, \gamma, S$ and $w_{k}(k=1,2, \cdots)$ are either constants or variables that have already been predetermined prior to the commencement of smoothing. In order to perform a comparison of the accuracies obtained from the two smoothing approaches, a numerical test is conducted to compute the outputs of $F(i)$. To test the effect of different SWF on the smoothing result, two sets of SWF are explored in this investigation. The first set allows SWF to decrease by 0.010 (i.e. $\delta w_{i}=0.010$ ) with the increase of epoch and the second set allows a decrease of $0.005\left(\delta w_{i}=0.005\right)$ each epoch. Corresponding to the first set of SWF, there are 100 epochs used in the smoothing (the number of used epochs is determined by $1 / \delta w_{i}$ ) and 200 epochs in second set of SWF.

The function outputs corresponding to the two sets of SWF are depicted in Figs. 1 and 2, respectively. In both figures, the $F(i)$ outputs and the standard deviations of TEC data smoothed by approach I and II are plotted. Figures 1 and 2 indicate that for both approaches I and II, the TEC data have better accuracies when more epochs of GPS measurements are included in smoothing. Both Figs. 1 and 2 show that the standard deviation of the smoothed TEC data inferred from approach I is inversely proportional to the number of GPS epoch. The standard deviations obtained from approach I show a steady and nearly linear trend of decrease with the increase of epochs. Compared to approach I, the standard deviations of TEC data derived from approach II decrease at an exponent mode. The standard deviations obtained from approach II decrease rapidly with the inclusion of epochs. Particularly during the first 20 epochs, a 


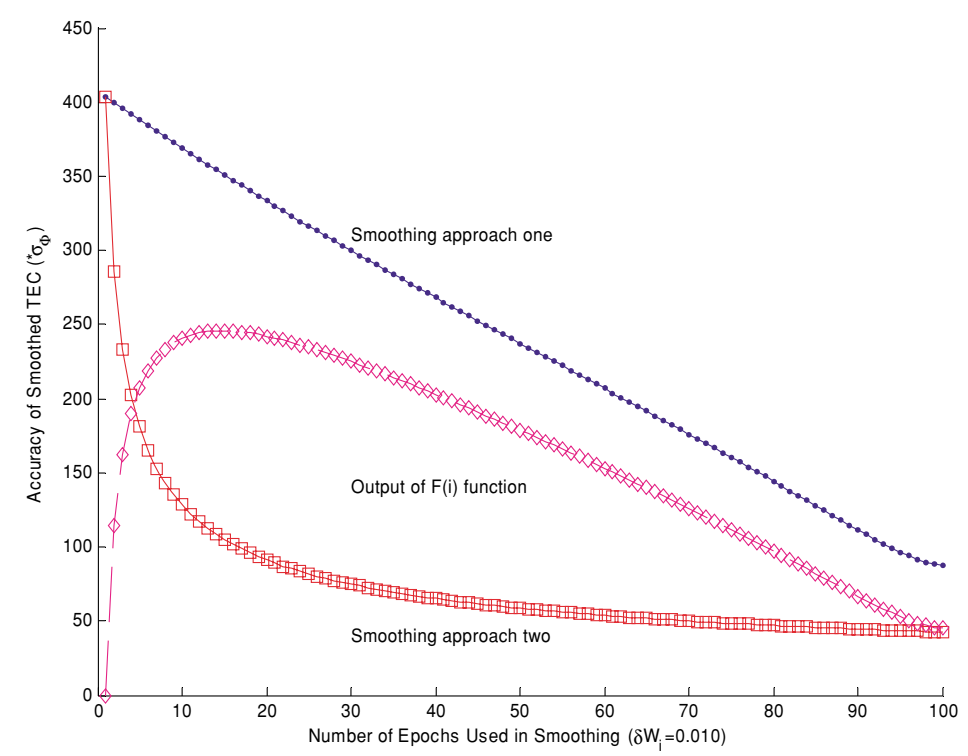

Fig. 1. Comparison of TEC accuracies from two smoothing approaches $\left(\delta W_{i}=0.010\right)$.

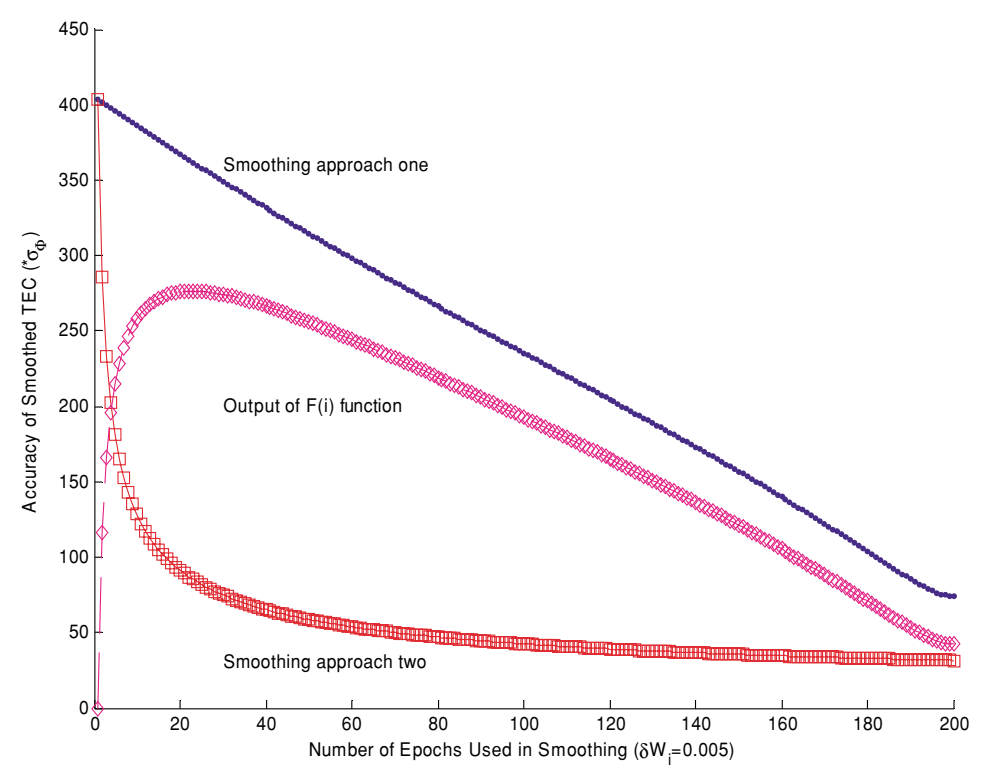

Fig. 2. Comparison of TEC accuracies from two smoothing approaches $\left(\delta W_{i}=0.005\right)$.

remarkable decrease is identified in the results of approach II. Both Figs. 1 and 2 show that the function $F(i)$ has a positive output value at each smoothing epoch. This implies that the TEC standard deviations obtained from approach II are always smaller than those from approach I. Both figures show that there is one epoch where $F(i)$ has a peak value. At this peak epoch, the accuracy difference between approaches I and II reaches a maximum. Figure 1 shows that at $i=15, F(i)$ has a maximum value of $245.8 \sigma_{\Phi}$. For Fig. 2, the maximum $F(i)$ output $276.4 \sigma_{\Phi}$ occurs at $i=23$. The peak values in Figure 1 and Figure 2 are equivalent to 0.25 TECU and 0.28 TECU if the carrier phase noise level is considered to be $1 \mathrm{~mm}$, i.e. $\sigma_{\Phi}=1 \mathrm{~mm}$.

The peak $F(i)$ value in Fig. 2 appears late than Fig. 1. This is because the SWF step size $\delta w_{i}$ in Fig. 2 is smaller than Fig. 1. This implies that more epochs of carrier phase measurements need to be included into the smoothing when $\delta w_{i}$ is smaller to achieve the same smoothing effect as the larger $\delta w_{i}$. Both Figs. 1 and 2 show that within the first approximately 25 epochs, the accuracy difference between approaches I and II is the biggest. After the epoch where peak $F(i)$ value occurs, the magnitude of accuracy improvement starts to decline in an almost linear fashion with the increase of smoothed epochs.

\section{Evaluation of Approximate Formula of Ap- proach I}

It should be pointed out that for smoothing approach I (i.e. Eq. (1)), some literatures used an approximate formula to estimate the standard deviations of the smoothed code pseudorange measurements (Cheng, 1999).

$$
\sigma_{R\left(t_{i}\right)_{\mathrm{sm}}}^{2}=w_{i}^{2} \sigma_{R\left(t_{1}\right)}^{2}+\left(1-w_{i}\right)^{2}\left(\sigma_{R\left(t_{i-1}\right)_{\mathrm{sm}}}^{2}+2 \sigma_{\Phi\left(t_{1}\right)}^{2}\right)
$$


The above formula is only an approximate formula to estimate the standard deviation of the smoothed code pseudorange measurement because the correlation of the twice used carrier phase measurements is not taken into account in Eq. (19). As Eq. (1) shows, carrier phase measurement $\Phi\left(t_{i-1}\right)$ at epoch $t_{i-1}$ is used in deriving the smoothed pseudorange $R\left(t_{i-1}\right)_{\mathrm{sm}}$. At the next epoch $t_{i}, \Phi\left(t_{i-1}\right)$ is used again. But in the approximate formula Eq. (19), the reuse of the same carrier phase measurement is not taken into account. Thus the formula obtained in Eq. (19) is incompact and the use of it will lead to plausible results, as be shown in the following test.

Starting from the initial condition $\sigma_{R\left(t_{1}\right)_{\mathrm{sm}}}^{2}=w_{1}^{2} \sigma_{R\left(t_{1}\right)}^{2}=$ $\sigma_{R\left(t_{1}\right)}^{2}=S \sigma_{\Phi}^{2}$, the expanded analytical expression for formula (19) can be derived as follow:

$$
\begin{aligned}
\sigma_{R\left(t_{i}\right)_{\mathrm{sm}}}= & \sigma_{\Phi}\left\{S w_{i}^{2}+\sum_{k=1}^{i-1}\left(S w_{k}^{2}+2\right)\left(1-w_{k+1}\right)^{2}\right. \\
& \left.\times\left(1-w_{k+2}\right)^{2} \cdots\left(1-w_{i}\right)^{2}\right\}^{1 / 2}
\end{aligned}
$$

Similar to Eq. (7), the standard deviation of the smoothed TEC data can be calculated by:

$$
\begin{aligned}
\sigma_{\mathrm{TEC}\left(t_{i}\right)}= & \frac{\sqrt{2} f_{1}^{2} \sigma_{\Phi\left(t_{1}\right)}}{40.3 \times 10^{16}(\gamma-1)} \\
& \times\left\{S w_{i}^{2}+\sum_{k=1}^{i-1}\left(S w_{k}^{2}+2\right)\left(1-w_{k+1}\right)^{2}\right. \\
& \left.\times\left(1-w_{k+2}\right)^{2} \cdots\left(1-w_{i}\right)^{2}\right\}^{1 / 2}
\end{aligned}
$$

To compare the difference between the approximate formula Eq. (19) and closed-form formula Eq. (7), a numerical test is conducted with different values of SWF $\left(\delta W_{i}=0.010\right.$ and $\left.\delta W_{i}=0.005\right)$ and different noise ratios (code pseudorange noise versus carrier phase noise) ( $S=100$ and $S=900$ ). The standard deviations computed from the two formulas are presented in Fig. 3 to Fig. 6. It can be seen that using the approximate formula, the variation of standard deviations of the smoothed TEC data demonstrates an abnormal divergence since the 91th epoch in Fig. $3\left(\delta W_{i}=0.010, S=100\right)$, the 99th epoch in Fig. $4\left(\delta W_{i}=0.010, S=900\right)$, the 178th epoch in Fig. $5\left(\delta W_{i}=0.005, S=100\right)$ and the 196th epoch in Fig. $6\left(\delta W_{i}=0.005, S=900\right)$. These epochs where the divergence occurs are referred to as "turning points" in this paper. Prior to the divergence at the turning points, the standard deviations generally show a linearly decreasing trend with the increment of number of epochs used in smoothing. Since the epoch at the turning points, this trend reverses. The standard deviation of the smoothed TEC data starts to show a trend of increase hereafter the turning point. This is somewhat contradictory to the common sense that the smoothed TEC data should have smaller standard deviation while more epochs are included in the smoothing. The reason that causes this abnormality is the incompact approximation implied in Eq. (19) as discussed above not taking the correlation into account. Figure 3 to Fig. 6 indicate that the approximate formula Eq. (19) is incompact and the use of it will lead to plausible result when the smoothing is performed beyond the turning points. In a comparison, the results obtained from Eq. (7), which is derived by strictly conforming to the error propagation law, are more reasonable.

The test results also indicate that smaller the code pseudorange to carrier phase noise ratio is, the earlier the turning point shows up in the smoothing. In Figs. 3 and 5 (both with $S=100$ ), the turning points appear at the 91th and 178th epoch, respectively, while in Figs. 4 and 6 (both with $S=900$ ), the turning points show up at the 99th and 196th epoch respectively. This implies that when using dual frequency GPS measurements of low code pseudorange to carrier phase noise ratio to derive smoothed TEC data, more cautions should be taken in the employment of approximate formula Eq. (19) to guard against the divergence of standard deviation after turning points.

The bottom subplots in Fig. 3 to Fig. 6 show the difference of standard deviations between the approximate formula and the closed-form one. The four subplots unanimously indicate that the accuracy derived from the closedform formula is better than that from the approximate formula. It shows that the accuracy difference is steadily increasing with the number of smoothed epochs, reaching a maximum difference at the end of smoothing. An examination of Fig. 3 to Fig. 6 reveals that the maximum accuracy difference varies with the value of $S$. As Fig. 3 to Fig. 6 show, the maximum accuracy differences computed with a small value of $S$ are much higher than those obtained with a large value of $S$. In Figs. 3 and. 5 (both with $S=100$ ), the maximum accuracy differences are $28.3 \sigma_{\Phi}$ and $40.9 \sigma_{\Phi}$ at the end of smoothing, respectively. For Figs. 4 and 6 (both with $S=900$ ), their maximum accuracy differences are $13.8 \sigma_{\Phi}$ and $23.1 \sigma_{\Phi}$ at the end of smoothing, respectively. The maximum accuracy differences in both Figs. 3 and 5 are much larger than those in Figs. 4 and 6. Furthermore, it can be found that with the same $S$, the maximum accuracy differences calculated with a small $\delta W_{i}$ have a higher value than those with a big $\delta W_{i}$. In Figs. 5 and 6 (both with $\delta W_{i}=0.005$ ), their maximum accuracy differences are $40.9 \sigma_{\Phi}$ and $23.1 \sigma_{\Phi}$ respectively, which are much higher than their counterparts Figs. 3 and 4 (both with $\left.\delta W_{i}=0.010\right)$, which have maximum accuracy differences of $28.3 \sigma_{\Phi}$ and $13.8 \sigma_{\Phi}$, respectively. An examination of the bottom subplots in Fig. 3 to Fig. 6 also finds that when the number of epochs used for smoothing is larger than approximately $70 \%$ of the total smoothing epochs $\left(1 / \delta w_{i}\right)$, the accuracy difference between the closed-form formula and the approximate one becomes to be increasing rapidly and the difference is no longer negligible.

The analysis reveals that the closed-form formula has apparent advantage over the approximate formula. The results indicate that it is necessary to consider the correlation of the common phase measurements in consecutive epochs when deriving standard deviation of smoothed pseudorange. The closed-form formula has taken the correlation into account and thus it demonstrates an advantage over the approximate one. This advantage becomes more ap- 

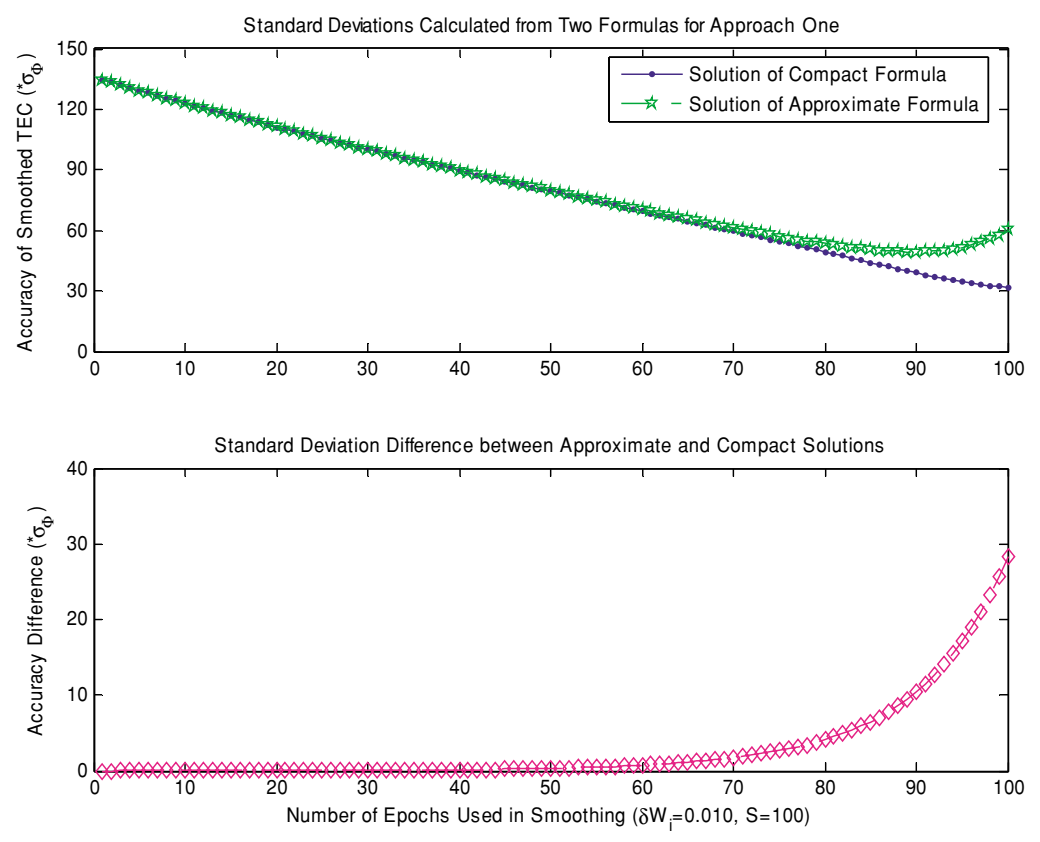

Fig. 3. Comparison of two methods of calculating standard deviations $\left(\delta W_{i}=0.010, S=100\right)$.
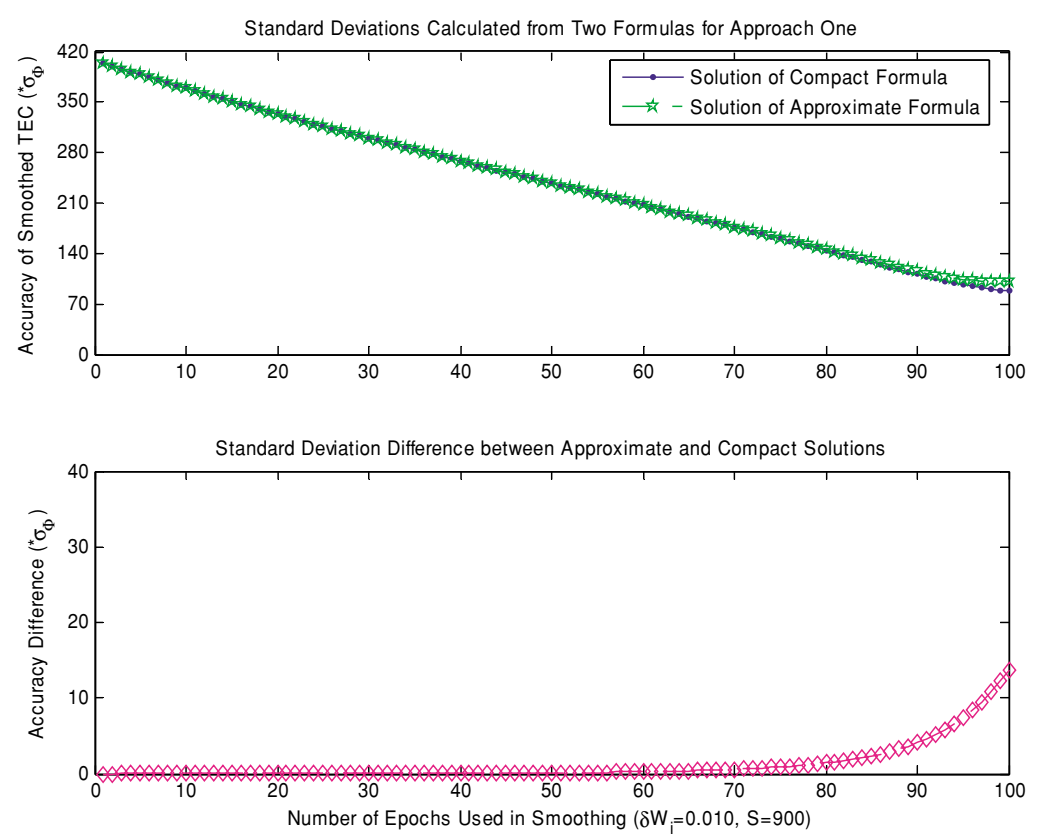

Fig. 4. Comparison of two methods of calculating standard deviations $\left(\delta W_{i}=0.010, S=900\right)$.

parent when the number of GPS measurement epochs used for smoothing increases. The magnitude of the superiority of the closed-form formula to the approximate one will become increasingly visible when 1) the number of epochs used for smoothing is large enough, approximately larger than $70 \%$ of the total smoothing epochs $\left(1 / \delta w_{i}\right)$; particularly when it approaches the turning point; 2 ) the value of $S$, code pseudorange to carrier phase noise ratio, is small; 3 ) the step size of SWF, (i.e. $\delta W_{i}$ ), is small. Under such three conditions, the use of approximate formula should be particularly avoided in order not to deteriorate the accuracies of the smoothed TEC data.

\section{Conclusion}

Two widely used smoothing approaches for deriving high precision TEC data from dual frequency GPS measurements have been investigated and their closed-form formulas for computing the precision of smoothed TEC data have been developed. The accuracies of the smoothed TEC data obtained from the two approaches have been compared through numerical analyses with different SWF schemes. The results indicate that the TEC data smoothed by approach II have better accuracies than those derived from approach I, especially when using small number of smoothing epochs (less than about 25 epochs).

For approach I, an approximate formula that was previ- 

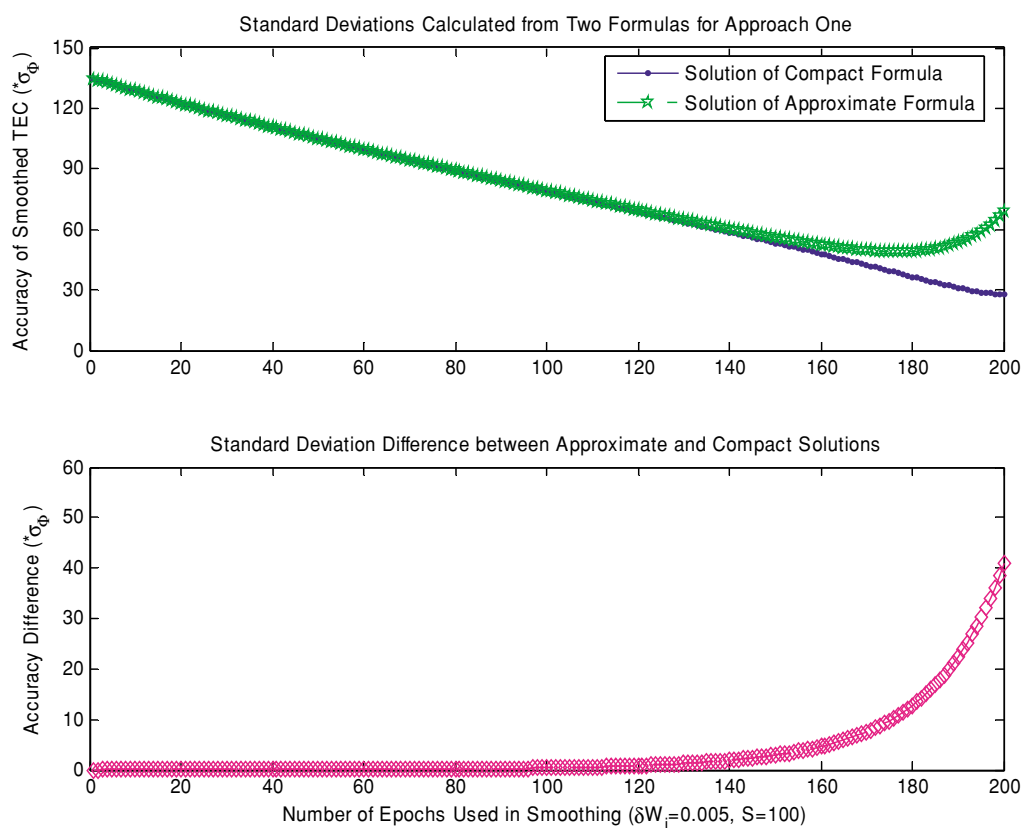

Fig. 5. Comparison of two methods of calculating standard deviations $\left(\delta W_{i}=0.005, S=100\right)$.
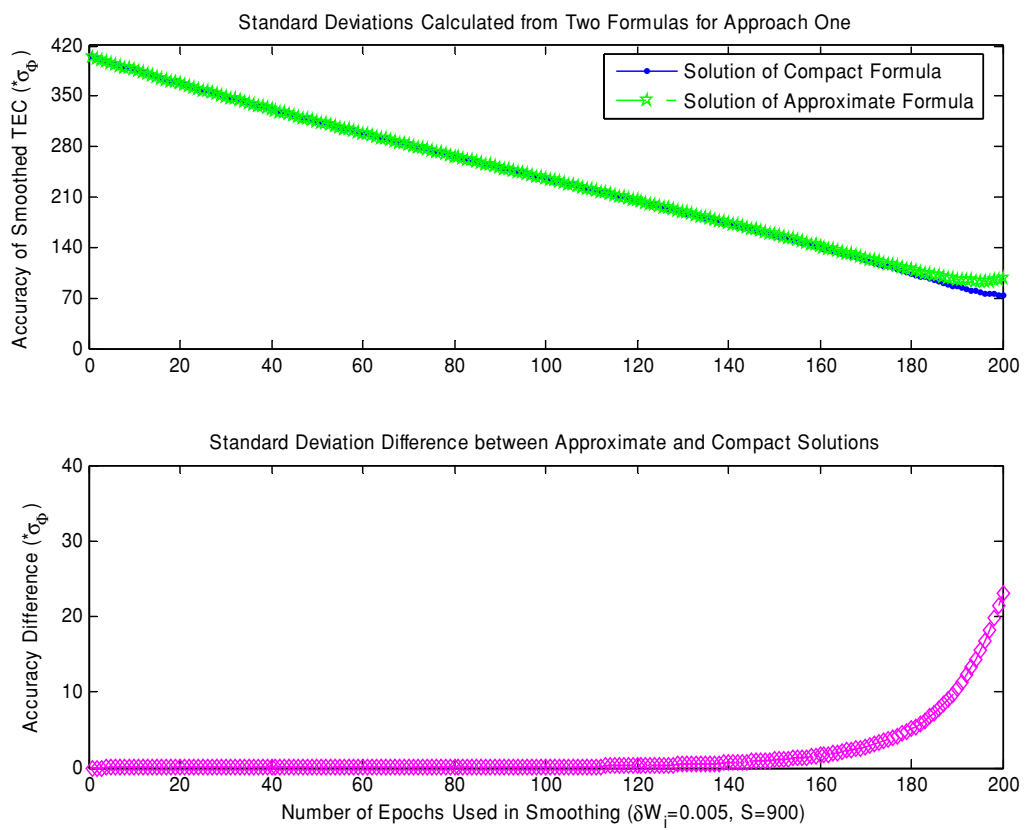

Fig. 6. Comparison of two methods of calculating standard deviations $\left(\delta W_{i}=0.005, S=900\right)$.

ously proposed to estimate the accuracy of smoothed code pseudorange measurements is also examined and the TEC data accuracies derived from the approximate formula have been compared to the closed-form formula. A divergence of the TEC precision is identified in the data analysis when using the approximate formula. The analysis reveals that there exists a turning point in the use of the approximate formula for accuracy estimation. After the turning point, the estimated precision starts to diverge. The data analysis indicates that the divergence becomes more severe when the ratio of pseudorange noise level to carrier phase one (the value of $S$ ) or the step size of SWF (the value of $\delta W_{i}$ ) becomes small.
Based on the numerical analysis results presented in this paper, the smoothing approach II should be used to derive the smoothed TEC data from dual frequency GPS measurements, particularly when only a short period of GPS data are available for smoothing. In the future work, the two smoothing approaches will be further studied with real GPS measurements and a comparison of smoothed TEC results obtained from the two approaches will be performed.

Acknowledgments. This research work has been partially supported by the projects from Natural Sciences and Engineering Research Council of Canada (NSERC) and Geomatics for Informed Decisions (GEOIDE), Canada. 


\section{References}

Cheng, P., Remarks on Doppler-aided smoothing of code ranges, Journal of Geodesy, 73, 23-28, 1999.

Hajj, G. A., R. Ibañez-Meier, E. R. Kursinski, and L. J. Romans, Imaging the ionosphere with the Global Positioning System, International Journal of Imaging Systems and Technology, 5(2), 174-184, 1994.

Hatch, R., Synergism of GPS code and carrier measurements, Proceedings of the Third International Geodetic Symposium on Satellite Doppler Positioning, 2, 1213-1231, New Mexico State University, NM, USA, Feb. 8-12, 1982.

Hatch, R., Dynamic differential GPS at the centimeter level, Proceedings of the 4th International Geodetic Symposium on Satellite Positioning, 2 , 1287-1298, Austin, TX, USA, Apr. 28-May 2, 1986.

Hernández-Pajares, M., J. M. Juan, and J. Sanz, New approaches in global ionospheric determination using ground GPS data, Journal of Atmospheric and Solar-Terrestrial Physics, 61, 1237-1247, 1999.

Hernández-Pajares, M., J. M. Juan, J. Sanz, and D. Bilitza, Combining GPS measurements and IRI model values for space weather specification, Advances in Space Research, 29(6), 949-958, 2002.

Jin, X. X., Theory of carrier adjusted DGPS positioning approach and some experimental results, PhD thesis, Delft University Press, Delft, 1996.

Lachapelle, G., J. Hagglund, W. Falkenberg, P. Bellemare, M. Casey, and M. Eaton, GPS land kinematic positioning experiments, Proceedings of the 4th International Geodetic Symposium on Satellite Positioning, 2, 1327-1344, Austin, TX, USA, Apr. 28-May 2, 1986.

Langley, R., M. Fedrizzi, E. de Paula, I. Kantor, M. Santos, and A. Komjathy, Mapping the low-latitude ionosphere with GPS, GPS World, 13(2), 41-47, 2002.
Leick, A., GPS Satellite Surveying, 560 pp., John Wiley, New York, 1995. Liu, Z. Z., Ionosphere tomographic modeling and applications using Global Positioning System (GPS) measurements, Ph.D thesis, 304 pp., Department of Geomatics Engineering, The University of Calgary, Calgary, Alberta, Canada, 2004.

Liu, Z. Z. and Y. Gao, Ionospheric tomography using GPS measurements, Proceedings of the International Symposium on Kinematic Systems in Geodesy, Geomatics and Navigation, 111-120, Banff, Alberta, Canada, Jun. 5-8, 2001.

Liu, Z. Z., S. Skone, Y. Gao, and A. Komjathy, Ionospheric modeling using GPS data, GPS Solutions, 9(1), DOI: 10.1007/s10291-004-0129z, 2005.

Sardon, E., A. Rius, and N. Zarraoa, Estimation of the transmitter and receiver differential biases and the ionospheric total electron content from Global Positioning System observations, Radio Science, 29, 577586, 1994.

Sekido, M., T. Kondo, E. Kawai, and M. Imae, Evaluation of GPS-based ionospheric TEC map by comparing with VLBI data, Radio Science, 38(4), 1069, doi:10.1029/2000RS002620, 2003.

Skone, S., Wide area ionosphere grid modeling in the auroral region, Ph.D thesis, Department of Geomatics Engineering, The University of Calgary, Calgary, Alberta, Canada, 1998.

Skone, S., V. Hoyle, S. Lee, and S. Poon, Variations in point positioning accuracies for single frequency GPS users during solar maximum, $\mathrm{Ge}$ omatica, 56(2), 131-140, 2002.

Z. Liu (e-mail: zzliu@ucalgary.ca), Y. Gao, and S. Skone 